\title{
Editorial (Special)
}

\section{Preface to Special Issue for Chinese Symposium on Photocatalytic Materials (CSPM)}

The Chinese Symposium on Photocatalytic Materials (CSPM) was held at the Wuhan University of Technology, Wuhan, China on July 26-29, 2018. The CSPM symposium was jointly organized by Wuhan University of Technology, Fuzhou University and Xuchang University, and was supported by National Natural Science Foundation of China (NSFC), Wuhan University of Technology, Fuzhou University, Xuchang University, Beijing Perfectlight and others. More than 320 colleagues from four continents (Asia, America, Australia and Europe) participated in this symposium, and presented 10 plenary lectures, 13 keynote lectures, 35 invited lectures, 8 oral lectures and 144 posters. 10 best posters were selected.

The purpose of this symposium is to provide an international forum for overseas Chinese and local experts in the photocatalytic field. It provides a platform to discuss the recent advancement and to facilitate scientific exchange. The CSPM symposium includes the following topics: fundamental research on photocatalytic materials, doping and bandgap engineering of photocatalytic materials, tuning morphology and facets of photocatalytic materials, strategies for photocatalytic material preparation, first-principle study of photocatalytic materials, environmental application of photocatalytic materials, photocatalytic water splitting for hydrogen and oxygen evolutions, photocatalytic $\mathrm{CO}_{2}$ reduction to solar fuels, photoelectrocatalysis, dye-sensitized, perovskite solar cells and other related research.

This special issue of Chinese Journal of Catalysis (CJC) contains 18 papers, including 2 review papers, 1 Communication and 15 research papers. Most of them were presented at the CSPM symposium. All the published papers were strictly peer reviewed according to normal review practices for the journal. As the Guest Editors, we would like to thank all the authors for contributing their high-quality papers and reviewers for reviewing the submitted manuscripts. Furthermore, we would like to thank the editorial staff of the journal for their kind assistance.

\section{Acknowledgments}

The CSPM symposium was financially supported by NSFC (U1705251, 51320105001 and 21433007).

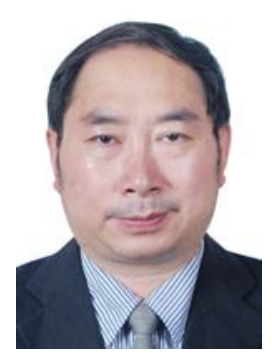

Prof. Jiaguo Yu (Guest Editor)

State Key Laboratory of Advanced Technology for Material Synthesis and Processing, Wuhan University of Technology, Wuhan, China E-mail: jiaguoyu@yahoo.com

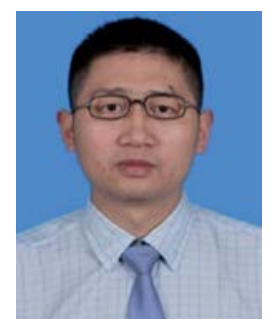

Prof. Xin Li (Guest Editor)

College of Forestry and Landscape Architecture, Key Laboratory of Energy Plants Resource and Utilization, Ministry of Agriculture, South China Agricultural University, Guangzhou, China

E-mail: Xinliscau@yahoo.com

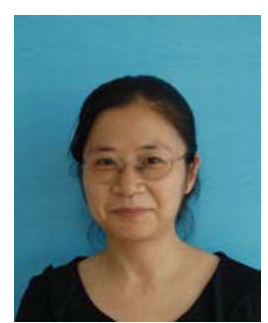

Prof. Zhaohui Li (Guest Editor)

State Key Laboratory of

Photocatalysis on Energy and Environment, College of Chemistry, Fuzhou University,

Fuzhou, China

E-mail: zhaohuili1969@yahoo.com

DOI: S1872-2067(19)63308-5 | http://www.sciencedirect.com/science/journal/18722067 | Chin. J. Catal., Vol. 40, No. 3, March 2019 (C) 2019, Dalian Institute of Chemical Physics, Chinese Academy of Sciences. Published by Elsevier B.V. All rights reserved. 